\title{
A Hybrid Algorithm Based on a Modified Sine Cosine Algorithm and Least Square and Its Application to Microwave Imaging
}

\author{
Meng Wang $(\mathbb{D})$ and Guizhen Lu $(\mathbb{D}$ \\ College of Information and Communication Engineering, Communication University of China, Beijing 100024, China \\ Correspondence should be addressed to Meng Wang; wangmeng1993@cuc.edu.cn
}

Received 15 December 2020; Revised 3 March 2021; Accepted 24 March 2021; Published 5 April 2021

Academic Editor: Mauro Parise

Copyright (C) 2021 Meng Wang and Guizhen Lu. This is an open access article distributed under the Creative Commons Attribution License, which permits unrestricted use, distribution, and reproduction in any medium, provided the original work is properly cited.

\begin{abstract}
With the rapid development of materials science and medical imaging technology, traditional optimization algorithms cannot solve the problem of inverse scattering of complex scatterers well. Therefore, more and more imaging algorithms for solving complex scatterers were proposed. In this paper, a novel hybrid algorithm is put forward for the microwave imaging problem. First, the proposed algorithm improves the search path of the traditional sine cosine algorithm, which obtains better global search capability. Second, the least square is introduced to form judging and contrasting mechanisms, which forms the parallel algorithm simultaneously, in order to make the proposed algorithm more suitable for the diverse microwave imaging problem. To prove the efficiency of the proposed algorithm, several examples, including ten benchmark functions, two engineering design problems, and three different microwave imaging problem tests are adopted. As expected, the results show that the proposed algorithm achieves not only superior optimal value but also the reconstruction of the complicated permittivity of the scatterer compared with several traditional optimization algorithms.
\end{abstract}

\section{Introduction}

The research of microwave imaging has been widely applied in medical imaging, underground detection, and other engineering fields [1-3]. However, such microwave imaging problems are nonlinear and ill-posed. Traditional methods are based on gradient information. Although the computational efficiency is high, it is easy to get the local optimum, instead of the global optimum. Especially, with the rapid development of the field of artificial metamaterials, the permittivity distribution of materials is becoming more and more complicated. Traditional methods based on gradient information cannot reconstruct the permittivity distribution of such scatterers well.

Traditional stochastic optimization algorithms have been widely used in microwave imaging [4-6]. Researchers transformed the traditional microwave imaging problem into an optimization problem and solved it through optimization algorithms. For instance, researchers [7-10] introduced particle swarm optimization (PSO) to solve the inverse scattering problems, and the results proved the feasibility of PSO. Researchers introduced the engineering application of PSO and differential evolution (DE) to reconstruct the shape of a perfect electrical conductor, human tissue, scatterer profile, and shape from the measurement data of the scattered field [11-13].

An improved evolution strategy was proposed, and it was applied to the microwave imaging. The advantages of the proposed algorithm were proved by the numerical results [14]. In [15], bat algorithm (BA), as a natural heuristic optimization algorithm with excellent performance, was used to solve microwave imaging problems. In [16], the whale optimization algorithm (WOA) was applied to solve the inverse scattering problem, which was a problem solving the reconstruction of an imperfect conductor with corners.

Although the stochastic optimization algorithm provides a new solution to the microwave imaging problem, there are still some performance problems, such as slower convergence rate and large error, when solving complicated microwave imaging problem, such as layered media and materials with bubbles. 
Researchers have been studying and exploring new stochastic optimization methods during these ten years. Many optimization algorithms with good performance have been proposed, such as the naked mole-rat (NMR) algorithm [17] and several improved whale optimization algorithms. The researchers [18] proposed a novel optimization algorithm, called the sine cosine algorithm (SCA). SCA has been widely applied in the engineering field due to its good performance [19-22]. The results of applications proved that SCA performed well because it had more flexible search path and very few algorithm parameters [23-25]. In the iterative process, it easily found the global optimum with high search efficiency. Meanwhile, different researchers have been constantly improving the SCA according to specific engineering problems and obtaining good performance [26-28].

To summarize, it is all known that there is currently no SCA method applied to microwave imaging. Furthermore, when traditional stochastic optimization algorithms are applied to solve microwave imaging problems with complicated scatterers, such as layered media and materials containing bubbles, accurate results cannot be obtained due to the high degree of nonlinearity and morbidity of the problem.

In this paper, a hybrid algorithm (HA) based on an improved sine cosine algorithm and least square is proposed. A novel parameter is introduced to improve the search path, so as to be more widely applied in complex inverse scattering problems.

To prove the property of the proposed algorithm, the benchmark function test, engineering design problems, and three microwave imaging problems are applied. The results are compared with that of the traditional metaheuristic algorithm, such as particle swarm optimization (PSO) [29], gray wolf optimizer (GWO), and moth-flame optimization (MFO). The results show that the proposed algorithm can overcome the shortcoming of being easily trapped in the local minimum and that it is more suitable for the complicated microwave imaging problem.

The composition of paper is arranged as follows. In Section 2, the mathematical model of the two-dimensional microwave imaging problem is described. In Section 3, the hybrid algorithm is introduced. In Section 4, the benchmark function tests, engineering design problems, and three different microwave imaging problems are applied to verify the property of the proposed hybrid algorithm (HA).

\section{Problem Description}

As shown in Figure 1, the incident field is generated by the incident antenna, and the scattered field of the scatterer can be received at the receiving line by the scattering effect of unknown objects in the detection area. The intensity of the scattered field is determined by the distribution of electromagnetic parameters of the scatterers in the imaging domain. Under the condition that the parameter distribution of the scatterer is known, the mathematical process of calculating the scattering field is the forward scattering of the electromagnetic field, and the process of calculating the parameter distribution of the object in the electromagnetic

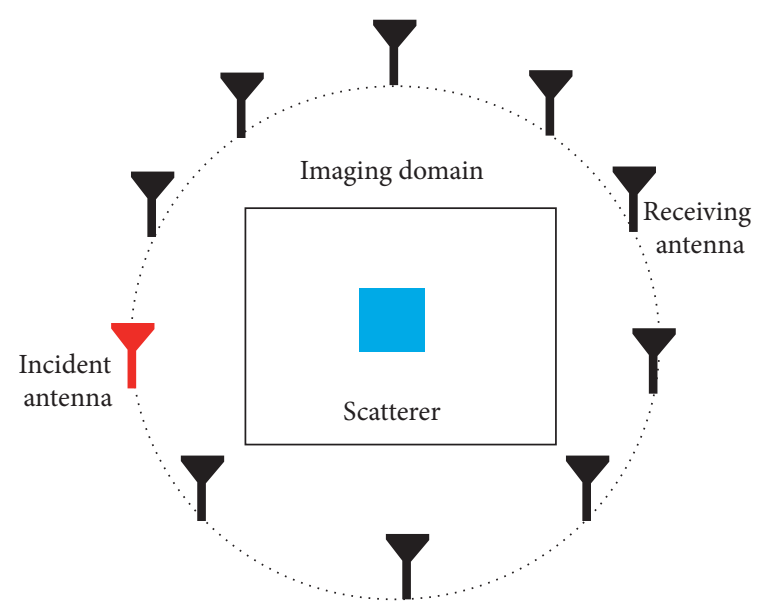

FIGURE 1: The simplified model of microwave imaging.

field through the scattering field at the receiving antenna is the inverse scattering problem [30].

In the process of solving this problem, an important relationship is used. The total field can be counted as the superposition of the incident field and the scattered field [30]:

$$
E_{\mathrm{tot}}=E_{\mathrm{inc}}+E_{\mathrm{sca}} \text {, }
$$

where $E_{\text {tot }}$ represents the total field, $E_{\text {inc }}$ represents the incident field, and $E_{\text {sca }}$ represents the scattered field.

As it is known, the incident field satisfies the following Helmholtz equation:

$$
\nabla^{2} E_{\text {inc }}+k_{0}^{2} E_{\text {inc }}=0
$$

where $k_{0}^{2}=\omega^{2} \varepsilon_{0} \mu_{0}$.

Then, the Dyadic Green function is used. The solution of the scattering field is as follows:

$$
E_{\mathrm{sca}}(r)=i \omega \mu_{0} \int_{D} G\left(r, r^{\prime}\right) J\left(r^{\prime}\right) d r^{\prime},
$$

where $D$ represents the imaging domain. When the incident wave vector lies in the plane of the two-dimensional object,

$$
G\left(r, r^{\prime}\right)=\frac{i}{4} H_{0}^{1}\left(k_{0}\left|r-r^{\prime}\right|\right) \text {. }
$$

Finally, the Lippman-Schwinger equation is obtained:

$$
E(r)=E_{\mathrm{inc}}(r)+i \omega \mu_{0} \int_{D} G\left(r, r^{\prime}\right) J\left(r^{\prime}\right) d r
$$

where $J\left(r^{\prime}\right)=-i \omega \varepsilon_{0}\left[\varepsilon_{r}\left(r^{\prime}\right)-1\right] E\left(r^{\prime}\right)$.

It is obvious that equation (5) is nonlinear and highly illposed. $\varepsilon_{r}$ is unknown.

\section{A Hybrid Algorithm}

The sine cosine algorithm (SCA) was proposed by Seyedali Mirjalili in 2016 [18]. More details of the proposed algorithm could be found in detail in Section 3 of [18]. The search path is shown as follows: 


$$
X_{i}^{t+1}=\left\{\begin{array}{l}
X_{i}^{t}+r_{1} \times \sin \left(r_{2}\right) \times\left|r_{3} P_{i}^{t}-X_{i}^{t}\right|, r_{4}<0.5 \\
X_{i}^{t}+r_{1} \times \cos \left(r_{2}\right) \times\left|r_{3} P_{i}^{t}-X_{i}^{t}\right|, r_{4} \geq 0.5
\end{array} .\right.
$$

Due to the serious morbidity and nonlinearity of equation (5), traditional SCA cannot solve the microwave imaging problem well, especially the complicated permittivity distribution (the specific performance results will be shown in Section 4).

Inspired from the PSO, the traditional search path has been improved. The improved search path is

$$
X_{i}^{k+1}=\left\{\begin{array}{l}
r_{1} X_{i}^{k}+c_{1} \omega\left(P_{\text {best }-i}^{k}-X_{i}^{k}\right)+c_{2} \omega\left(G_{\text {best }}^{k}-X_{i}^{k}\right) \\
r_{1} X_{i}^{k}+c_{1} \omega\left(P_{\text {best }-i}^{k}-X_{i}^{k}\right)+c_{2} \omega\left(G_{\text {best }}^{k}-X_{i}^{k}\right)
\end{array},\right.
$$

where $\omega=\left\{\begin{array}{l}\sin \left(r_{2}\right), r_{3}<0.5 \\ \cos \left(r_{2}\right), r_{3}>0.5\end{array}, X_{i}^{k}\right.$ is the current position of the particle, $P_{\text {best }}^{k}$ is individual extreme, $G_{\text {best }}^{k}$ is global extremum, $r_{1}$ is the convergence factor, $c_{1}$ and $c_{2}$ are constants, and $\omega$ is the trigonometric coefficient.

In the improvement of the search path, the search paths of sine and cosine are retained to keep the search feature of the sine and cosine dual path. The absolute value in the traditional path is cancelled to make the search range larger, which is conducive to finding the global optimum. In addition, global variable $G_{\text {best }}$ is introduced into the path at the same time. Under the common constraints of global variable $G_{\text {best }}$ and individual variable $P_{\text {best }}$, the global optimum can be better found.

Through the improvement above, the search range will rapidly narrow towards the global optimum. The search will follow a smoother path to find the global best point, which is shown in Figure 2.

In [31, 32], a novel parameter called empirical optimal parameter $E_{\text {best }}$ was introduced into the search path. It had been successfully used into the antenna pattern optimization and the good performance had been obtained. Therefore, in the improved search path, empirical parameter $E_{\text {best }}$ is also introduced. The definition of $E_{\text {best }}$ can be found in [31, 32]. Finally, the final search path is shown as follows:

$$
X_{i}^{k+1}=\left\{\begin{array}{l}
r_{1} X_{i}^{k}+c_{1} \omega\left(P_{\text {best }-i}^{k}-X_{i}^{k}\right)+c_{2} \omega\left(G_{\text {best }}^{k}-X_{i}^{k}\right)+c_{3} \omega\left(E_{\text {best }}^{k}-X_{i}^{k}\right) \\
r_{1} X_{i}^{k}+c_{1} \omega\left(P_{\text {best }-i}^{k}-X_{i}^{k}\right)+c_{2} \omega\left(G_{\text {best }}^{k}-X_{i}^{k}\right)+c_{3} \omega\left(E_{\text {best }}^{k}-X_{i}^{k}\right)
\end{array},\right.
$$

where $\omega=\left\{\begin{array}{l}\sin \left(r_{2}\right), r_{3}<0.5 \\ \cos \left(r_{2}\right), r_{3}>0.5\end{array}\right.$.

In equation (8), $c_{1}, c_{2}$, and $c_{3}$ are constants. $r_{2} \in[0, \pi / 2]$, and $r_{3}$ is a random number.

The correct setting of the convergence factor plays a very important role in an optimization algorithm. The convergence factor is also improved in equation (8), which is defined as follows:

$$
r_{1}=r_{\max }-\left(r_{\max }-r_{\min }\right) \frac{t}{T}
$$

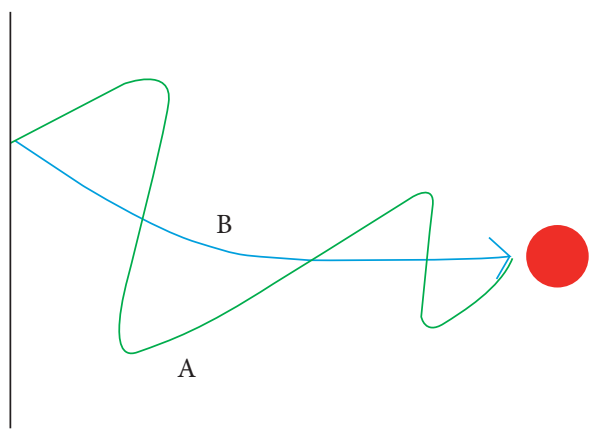

$$
\begin{aligned}
& \text { A: Traditional range change } \\
& \text { B: Improved range change }
\end{aligned}
$$

Figure 2: Traditional and improved range change.

where $r_{\max }$ and $r_{\min }$ are constants.

Diversified parameter settings can make the optimization algorithm more widely applicable to engineering problems.

The least square is a traditional mathematical optimization technique and is a deterministic method. It is all known that the single stochastic optimization algorithm is not suitable for microwave imaging of complicated scatterer. To make the algorithm more suitable for a wider range of microwave imaging problems, the least square method is combined with the modified SCA to form a hybrid and parallel algorithm. Through the combination of traditional stochastic optimization algorithms and deterministic algorithms and parallel computing, the proposed hybrid algorithm can adapt to more diverse electromagnetic field inverse problems. The flowchart of the hybrid algorithm is presented in Figure 3.

The specific process of the algorithm is as follows:

Step 1. First, the initial population is generated, that is, the relative permittivity is generated according to the number of blocks $N$ into which the scatterer is divided and the upper and lower bounds are searched by the algorithm. Step 2. The generated relative permittivity is brought into the objective function (fitness function), and the first objective function value is calculated. The scattering data of the forward electromagnetic field will be calculated according to the method of moments (MoM).

Step 3. The algorithm iteration starts; as the convergence factor continues to shrink, the search range for the relative permittivity gradually becomes smaller, and the boundary violation is handled to prevent the algorithm from crossing the boundary.

Step 4. The algorithm continues to iterate. After reaching the maximum number of iterations, the iteration is stopped and the relative permittivity gbest 1 generated by the iteration is outputted.

Step 5. In the process of steps (2)-(4), the least square method is used at the same time to obtain another set of relative permittivity gbest 2 . 


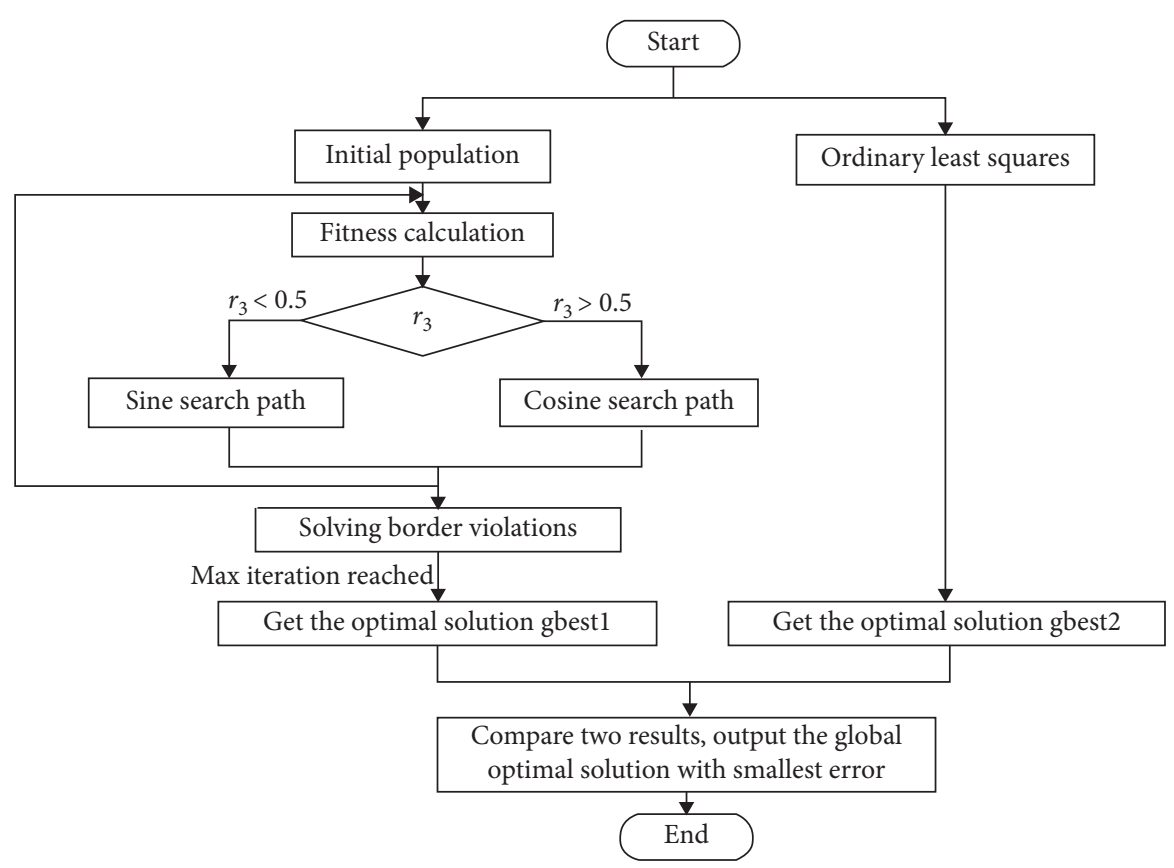

FIgURE 3: A flowchart of the hybrid algorithm.

Step 6. gbest1 and gbest 2 are substituted into the original scattering field solution formula, the error is calculated, the relative permittivity is selected with the smallest error to output, and the result is obtained.

\section{Experimental Results and Comparisons}

In this part, to measure the performance of the proposed method, ten benchmark functions which are chosen from [18], two engineering design, and three inverse scattering problems are applied. Microwaving imaging problems include the reconstructions of the uniform permittivity distribution, the layered permittivity distribution, and the materials with bubbles. All the data, the parameters of the listed algorithms and the settings of benchmark functions can be found in the following part.

4.1. Benchmark Functions Test. The performance of proposed algorithm is compared with traditional optimization algorithms genetic algorithm (GA), DE, PSO, moth-flame optimization (MFO), gray wolf optimizer (GWO), and SCA. The parameters of the algorithm settings are shown in Table 1.

The details of the benchmark functions are shown in Table 2 .

The optimal function values are listed in Table 3.

Based on the results and considering all of the benchmark function test results, for the benchmark function "F5," the proposed hybrid algorithm (HA) cannot get the better solution than the others, and for the function F10, the proposed HA cannot get the better solution as DE within the tolerance of error. For the other functions, the proposed algorithm is more efficient and accurate at solving the benchmark function tests, and it can obtain the superior optimal solution than the traditional algorithms listed.
TABLE 1: The parameter setting of algorithm.

\begin{tabular}{|c|c|c|}
\hline Algorithm & Parameter settings & $N$ \\
\hline PSO & $\begin{array}{c}\mathrm{Wmax}=0.9 \\
\mathrm{Wmin}=0.4 \\
c 1=0.5 \\
c 2=0.5\end{array}$ & 30 \\
\hline GA & $\begin{array}{c}\mathrm{Pc}=0.8 \\
\mathrm{Pm}=0.2 \text { gap }=0.9\end{array}$ & 30 \\
\hline $\mathrm{DE}$ & $\begin{array}{c}\text { Mutation rate: } F 0=0.5 \\
\text { Cross probability: } C R=0.9\end{array}$ & 30 \\
\hline GWO & The same as [33] & 30 \\
\hline MFO & The same as [34] & 30 \\
\hline SCA & $a=2$ & 30 \\
\hline Proposed algorithm & $\begin{array}{c}r_{\max }=0.9 \\
r_{\min }=0.4 \\
c 1=c 2=0.5\end{array}$ & 30 \\
\hline
\end{tabular}

4.2. Tension/Compression Spring Design Problem. Figure 4 shows the sketch map of this problem. It is a classic problem of verifying the performance of the proposed optimization algorithms. There are three variables in this problem: $D, P$, and $d$.

This problem is described in mathematical language as follows:

Consider $X=\left[\begin{array}{lll}x_{1} & x_{2} & x_{3}\end{array}\right]=\left[\begin{array}{lll}d & D & P\end{array}\right]$

Minimize $f(X)=\left(x_{3}+2\right) x_{2} x_{1}^{2}$

Subject to $g_{1}(X)=1-x_{2}^{3} x_{3} / 71785 x_{1}^{4} \leq 0 g_{2}(X)=4 x_{2}^{2}-$ $x_{1} x_{2} / 12566 \quad\left(x_{2} x_{1}^{3}-x_{1}^{4}\right)+1 / 5108 x_{1}^{2} \leq 0 g_{3}(X)=1-$ $140.45 x_{1} / x_{2}^{2} x_{3} \leq 0 \quad g_{4}(X)=x_{1}+x_{2} / 1.5-1 \leq 0$, where $0.05 \leq x_{1} \leq 2,0.25 \leq x_{2} \leq 1.30$, and $2.00 \leq x_{3} \leq 15$.

The detailed description of the problem can be found in [35]. The optimization results of all algorithms used are 
TABLE 2: The setting of benchmark functions.

\begin{tabular}{|c|c|c|c|c|}
\hline & Function & Dim & Range & $f_{\min }$ \\
\hline \multirow{4}{*}{ Unimodal functions } & $f_{1}(x)=\sum_{i=1}^{n} x_{i}^{2}$ & 10 & $x \in[-100,100]$ & 0 \\
\hline & $f_{2}(x)=\sum_{i=1}^{n}\left|x_{i}\right|+\prod_{i=1}^{n}\left|x_{i}\right|$ & 10 & $x \in[-10,10]$ & 0 \\
\hline & $f_{3}(x)=\sum_{i=1}^{n}\left(\sum_{j-1}^{i} x_{j}\right)^{2}$ & 10 & $x \in[-100,100]$ & 0 \\
\hline & $f_{4}(x)=\max _{i}\left\{\left|x_{i}\right|, 1 \leq i \leq n\right\}$ & 10 & $x \in[-100,100]$ & 0 \\
\hline \multirow{2}{*}{ Multimodal functions } & $f_{5}(x)=\sum_{i=1}^{n}-x_{i} \sin \left(\sqrt{\left|x_{i}\right|}\right)$ & 10 & $x \in[-500,500]$ & $-418.9829 \times 5$ \\
\hline & $f_{6}(x)=\sum_{i=1}^{n}\left[x_{i}^{2}-10 \cos \left(2 \pi x_{i}\right)+10\right]$ & 10 & $x \in[-5.12,5.12]$ & 0 \\
\hline \multirow{3}{*}{ Multimodal functions } & $f_{7}(x)=-20 \exp \left(-0.2 \sqrt{(1 / n) \sum_{i=1}^{n} x_{i}^{2}}\right)$ & 10 & $x \in[-32,32]$ & 0 \\
\hline & $-\exp \left((1 / n) \sum_{i=1}^{n} \cos \left(2 \pi x_{i}\right)\right)+20+e$ & & & \\
\hline & $f_{8}(x)=(1 / 400) \sum_{i=1}^{n} x_{i}^{2}-\prod_{i=1}^{n} \cos \left(x_{i} / \sqrt{i}\right)+1$ & 10 & $x \in[-600,600]$ & 0 \\
\hline \multirow{2}{*}{$\begin{array}{l}\text { Fixed-dimensions multimodal } \\
\text { benchmark function }\end{array}$} & $f_{9}(x)=\left((1 / 500)+\sum_{j=1}^{25} 1 / j+\sum_{i=1}^{2}\left(x_{i}-a_{i j}\right)^{6}\right)^{-1}$ & 2 & $x \in[-65.536,65.536]$ & 1 \\
\hline & $f_{10}(x)=\sum_{i=1}^{11}\left[a_{i}-\left(x_{1}\left(b_{i}^{2}+b_{i} x_{2}\right) / b_{i}^{2}+b_{i} x_{3}+x_{4}\right)\right]^{2}$ & 4 & $x \in[-5,5]$ & 0.0003 \\
\hline
\end{tabular}

TABLe 3: Optimal values of the benchmark functions.

\begin{tabular}{|c|c|c|c|c|c|c|c|}
\hline $\mathrm{F}$ & GA & $\mathrm{DE}$ & PSO & MFO & GWO & SCA & Proposed algorithm \\
\hline F1 & 5.0726 & $9.6466 \times 10^{-12}$ & 120.3183 & $6.0858 \times 10^{-14}$ & $8.5943 \times 10^{-58}$ & $6.6230 \times 10^{-16}$ & $2.1488 \times 10^{-71}$ \\
\hline $\mathrm{F} 2$ & 25.2404 & $1.8883 \times 10^{-6}$ & 5.2907 & $1.4231 \times 10^{-8}$ & $7.6446 \times 10^{-33}$ & $5.1195 \times 10^{-9}$ & $2.1747 \times 10^{-35}$ \\
\hline F3 & $1.0249 \times 10^{3}$ & $7.6280 \times 10^{-4}$ & 653.9996 & 0.4037 & $1.2557 \times 10^{-30}$ & $1.0708 \times 10^{-6}$ & $3.7612 \times 10^{-57}$ \\
\hline $\mathrm{F} 4$ & 27.4603 & 0.0026 & 13.8358 & 0.6668 & $2.6329 \times 10^{-19}$ & $7.1505 \times 10^{-5}$ & $5.0888 \times 10^{-21}$ \\
\hline F5 & -26.4461 & $-3.6354 \times 10^{3}$ & $-2.9342 \times 10^{3}$ & $-3.8330 \times 10^{3}$ & $-2.3731 \times 10^{3}$ & $-2.0143 \times 10^{3}$ & $-4.1898 \times 10^{3}$ \\
\hline F6 & 80.9664 & 16.8237 & 12.1527 & 30.8437 & 0.0000 & $9.5213 \times 10^{-13}$ & 0.0000 \\
\hline F7 & 19.9361 & $2.3264 \times 10^{-6}$ & 10.8616 & $1.4952 \times 10^{-7}$ & $7.9936 \times 10^{-15}$ & $6.6704 \times 10^{-8}$ & $8.8818 \times 10^{-16}$ \\
\hline F8 & 2.2488 & 0.5056 & 3.0373 & 0.1648 & 0.0610 & $2.1087 \times 10^{-10}$ & 0.0000 \\
\hline F9 & 4.9505 & 0.9980 & 8.8408 & 2.9821 & 2.9821 & 0.9995 & 0.9980 \\
\hline F10 & 0.0012 & 0.0003 & 0.0216 & 0.0008 & $3.7189 \times 10^{-4}$ & $7.1787 \times 10^{-4}$ & $3.1038 \times 10^{-4}$ \\
\hline
\end{tabular}

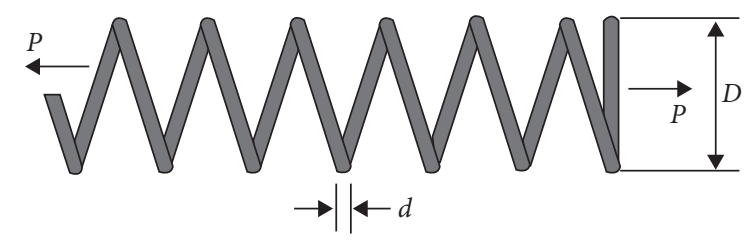

Figure 4: The model of tension/compression spring design problem.

shown in Table 4, which are from [35] (more details can be found in the network link https:/e-tarjome.com/storage/ panel/fileuploads/2019-08-22/1566462251_E11587-e-tarjome. pdf).

From the optimization results, it can be seen that the proposed hybrid algorithm (HA) can achieve the best optimal cost compared with the other algorithm listed.

4.3. Pressure Vessel Design Problem. Figure 5 shows the sketch map of this problem.

This problem is described in mathematical language as follows:

$$
\text { Consider } X=\left[\begin{array}{llll}
x_{1} & x_{2} & x_{3} & x_{4}
\end{array}\right]=\left[\begin{array}{llll}
T_{s} & T_{h} & R & L
\end{array}\right]
$$

Minimize $f(X)=0.6224 x_{1} x_{3} x_{4}+1.7781 x_{2} x_{3}^{2}+3.1661$ $x_{1}^{2} x_{4}+19.84 x_{1}^{2} x_{3}$

Subject to $g_{1}(X)=-x_{1}+0.00193 x_{3} \leq 0 g_{2}(X)=-x_{3}+$ $0.00954 x_{3} \leq 0 g_{3}(X)=-\pi \quad x_{3}^{2} x_{4}-4 / 3 \pi x_{3}^{2}+129600 \leq 0 g_{4}$ $(X)=x_{4}-240 \leq 0$, where $0 \leq x_{1}, x_{2} \leq 99,10 \leq x_{3}, x_{4} \leq 200$.

The detailed description of the problem can be found in [35]. All the results can be found in Table 5, which are from [35] (more details can be found in the network link https://e-tarjome.com/storage/panel/fileuploads/2019-08-22/ 1566462251_E11587-e-tarjome.pdf).

From the optimization results, it can be seen that the proposed hybrid algorithm (HA) can achieve the best optimal cost compared with the other algorithm listed. 
TABLE 4: The experimental results of HA and other algorithms for tension/compression spring design problem.

\begin{tabular}{|c|c|c|c|c|}
\hline \multirow{2}{*}{ Algorithm } & \multicolumn{3}{|c|}{ Optimal values for variables } & \multirow{2}{*}{ Optimal cost } \\
\hline & $\mathrm{d}$ & $\mathrm{D}$ & $P$ & \\
\hline WOA (Mirjalili and Lewis, 2016) & 0.051207 & 0.345215 & 12.54854 & 0.0126763 \\
\hline MVO (Mirjalili et al., 2016) & 0.05251 & 0.37602 & 10.33513 & 0.012790 \\
\hline CPSO (He and Wang, 2007) & 0.051728 & 0.357644 & 11.244543 & 0.0126747 \\
\hline GSA (Rashedi et al., 2009) & 0.0500 & 0.317312 & 14.22867 & 0.0128739 \\
\hline GA (Coello and Mezura-montes, 2002) & 0.051989 & 0.363965 & 10.890522 & 0.012681 \\
\hline ES (Mezura-montes and Coello, 2008) & 0.051643 & 0.3556 & 11.397926 & 0.012698 \\
\hline HS (Mahdavi et al, 2007) & 0.051154 & 0.349871 & 12.076432 & 0.0126706 \\
\hline CGDA (Baykasoğlu, 2012) & 0.0516925 & 0.3568108 & 11.2835059 & 0.012665 \\
\hline BA (Gandomi et al., 2013) & 0.05169 & 0.35673 & 11.2885 & 0.0126652 \\
\hline MFO (Mirjalili, 2015) & 0.051994457 & 0.36410932 & 10.868421862 & 0.0126669 \\
\hline HPSODE (Liu et al., 2010) & 0.0516888101 & 0.3567117001 & 11.289319935 & 0.0126652329 \\
\hline CDE (Hang et al., 2007) & 0.051609 & 0.354714 & 11.410831 & 0.0126702 \\
\hline UABC (Brajevic and Tuba, 2013) & 0.051691 & 0.356769 & 11.285988 & 0.012665 \\
\hline AFA (Baykasoğlu and Ozsoydan, 2015) & 0.051667 & 0.356198 & 11.319561 & 0.0126653 \\
\hline CSA (Askarzadeh, 2016) & 0.051689 & 0.356717 & 11.289012 & 0.0126652 \\
\hline TEO (Kaveh and Dadras, 2017) & 0.051775 & 0.358792 & 11.168390 & 0.012665 \\
\hline GWO (Mirjalili et al., 2014) & 0.05169 & 0.356737 & 11.28885 & 0.012665 \\
\hline EEGWO (Long et al., 2018) & 0.051673 & 0.35634 & 11.3113 & 0.012665 \\
\hline SCA (Mirjalili, 2016) & 0.05078 & 0.334779 & 12.72269 & 0.0127097 \\
\hline ISCA (Wen Long et al, 2018) & 0.0520217 & 0.364768 & 10.8323 & 0.012667 \\
\hline ROL-GWO (Wen Long et al, 2019) & 0.0517234 & 0.357538 & 11.2416 & 0.012666 \\
\hline HA (this work) & 0.0513 & 0.3184 & 10.1900 & 0.010214 \\
\hline
\end{tabular}

https://e-tarjome.com/storage/panel/fileuploads/2019-08-22/1566462251_E11587-e-tarjome.pdf.

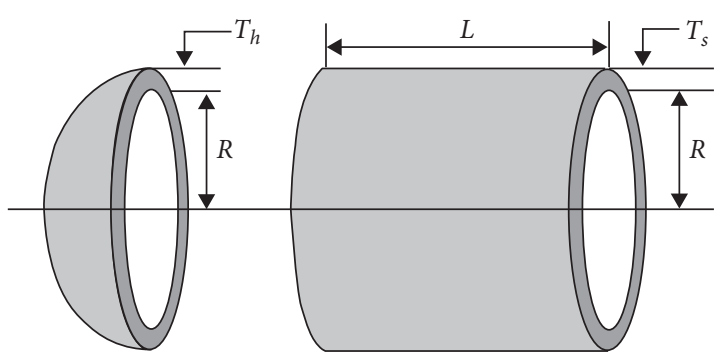

Figure 5: The model of pressure vessel design problem.

\subsection{Reconstruction of the Uniform Permittivity Distribution.} As an example, consider a problem of microwave imaging for the scatterer with uniform permittivity. The shape of scatterer is a square. The permittivity is 3 . The center coordinate of the conductor is located at $(0,0)$. The scatterer is separate into pieces of $4 \times 4(\operatorname{Dim}=16)$.

The transmit antenna with a frequency of $3 \mathrm{GHz}$ is adopted in the field model. The distance between the incentive source and the center of the scatterer is $0.5 \mathrm{~m}$. Sixty receiving antennas are evenly applied, with a radius of $0.5 \mathrm{~m}$, just as shown in Figure 6.

The fitness function is defined as

$$
f=\frac{\sum_{l=1}^{L} \sum_{m=1}^{M}\left|E_{s}^{l}\left(\overrightarrow{r_{m}}\right)-E_{m}^{l}\left(\overrightarrow{r_{m}}\right)\right|}{\sum_{l=1}^{L} \sum_{m=1}^{M}\left|E_{m}^{l}\left(\overrightarrow{r_{m}}\right)\right|}
$$

where $E_{m}^{\mathrm{l}}$ is the given scattered field data, which is calculated by method of moments (MoM), and $E_{s}^{l}$ is calculated by the algorithm.
In addition, the mean squared error (MSE) is also applied to judge the performance of the HA. It is defined as

$$
\mathrm{MSE}=\frac{1}{n} \sum_{i=1}^{n}\left[\varepsilon_{i}-\varepsilon_{r}\right]^{2},
$$

where $\varepsilon_{i}$ is the optimal solution, which is obtained by the proposed algorithm, and $\varepsilon_{r}$ equals 3 , which represents the known permittivity.

The results are listed in Table 6. These parameters are to judge the constancy of the results.

The convergence curve is shown in Figure 7.

Based on the results, the convergence rate of PSO is very fast. However, it is obvious that it is caught in the algorithm precocity. And, the stability of the DE solution is not better than the proposed HA. To sum up, considering the stability of the solution, the error of solution and the MSE, the proposed algorithm represents better than the other three optimization algorithms listed.

4.5. Reconstruction of Layered Permittivity Distribution. As an example, consider a microwave imaging problem for the scatterer with the layered permittivity distribution. The shape of the scatterer is the same as above, which has a layered relative permittivity distribution, as illustrated in Figure 8 . The permittivity is 2,8 , and 4 from the top to bottom in turn.

Four incentive sources with a frequency of $2 \mathrm{GHz}$ are adopted, $0.1 \mathrm{~m}$ from the center of the scatterer. Sixty receiving antennas are evenly applied, with a radius of $1.5 \mathrm{~m}$. 
TABLE 5: The experimental results of HA and other algorithms for pressure vessel design problem.

\begin{tabular}{|c|c|c|c|c|c|}
\hline \multirow{2}{*}{ Algorithm } & \multicolumn{4}{|c|}{ Optimal values for variables } & \multirow{2}{*}{ Optimal cost } \\
\hline & Ts & Th & $\mathrm{R}$ & $\mathrm{L}$ & \\
\hline WOA (Mirjalili and Lewis, 2016) & 0.8125 & 0.4375 & 42.0982699 & 176.638998 & 6059.7410 \\
\hline MVO (Mirjalili et al., 2016) & 0.8125 & 0.4375 & 42.090738 & 176.73869 & 6060.8066 \\
\hline CPSO (He and Wang, 2007) & 0.8125 & 0.4375 & 42.091266 & 176.7465 & 6061.0777 \\
\hline GSA (Rashedi et al., 2009) & 1.125 & 0.625 & 55.9886598 & 84.4542025 & 8538.8359 \\
\hline GA (Coello and Mezura-montes, 2002) & 0.8125 & 0.4375 & 42.097398 & 176.65405 & 6059.9463 \\
\hline ES (Mezura-montes and Coello, 2008) & 0.8125 & 0.4375 & 42.098087 & 176.640518 & 6059.7456 \\
\hline ACO (Kaveh and Talatahari, 2010) & 0.8125 & 0.4375 & 42.098353 & 176.637751 & 6059.7258 \\
\hline CGDA (Baykasoğlu, 2012) & 0.8125 & 0.4375 & 42.0975 & 176.6484 & 6059.8391 \\
\hline CSA (Gandomi et al., 2013) & 0.8125 & 0.4375 & 42.0984 & 176.6366 & 6059.7140 \\
\hline BA (Gandomi et al., 2013) & 0.8125 & 0.4375 & 42.0984 & 176.6366 & 6059.7143 \\
\hline MFO (Mirjalili, 2015) & 0.8125 & 0.4375 & 42.098445 & 176.636596 & 6059.7143 \\
\hline HPSODE (Liu et al., 2010) & 0.8125 & 0.4375 & 42.098446 & 176.636596 & 6059.714335 \\
\hline CDE (Hang et al., 2007) & 0.8125 & 0.4375 & 42.0984 & 176.6376 & 6059.7340 \\
\hline UABC (Brajevic and Tuba, 2013) & 0.8125 & 0.4375 & 42.098446 & 176.636596 & 6059.714335 \\
\hline AFA (Baykasoğlu and Ozsoydan, 2015) & 0.8125 & 0.4375 & 42.0984 & 176.6366 & 6059.7143 \\
\hline CSA (Askarzadeh, 2016) & 0.8125 & 0.4375 & 42.0984 & 176.6366 & 6059.7144 \\
\hline TEO (Kaveh and Dadras, 2017) & 0.8125 & 0.4375 & 42.0984 & 176.6366 & 6059.71 \\
\hline GWO (Mirjalili et al., 2014) & 0.8125 & 0.4375 & 42.0989181 & 176.758731 & 6051.5639 \\
\hline EEGWO (Long et al., 2018) & 13.09291 & 6.792196 & 42.09758 & 176.6495 & 6059.8704 \\
\hline SCA (Mirjalili, 2016) & 0.817577 & 0.417932 & 41.74939 & 183.5727 & 6137.3724 \\
\hline ISCA (Wen Long et al, 2018) & 12.96419 & 7.150134 & 42.09829 & 176.6392 & 6059.7489 \\
\hline ROL-GWO (Wen Long et al, 2019) & 12.73387 & 6.781898 & 42.09825 & 176.6397 & 6059.7528 \\
\hline HA (this work) & 0.8125 & 0.4375 & 42.0984 & 174.6670 & 6003.6540 \\
\hline
\end{tabular}

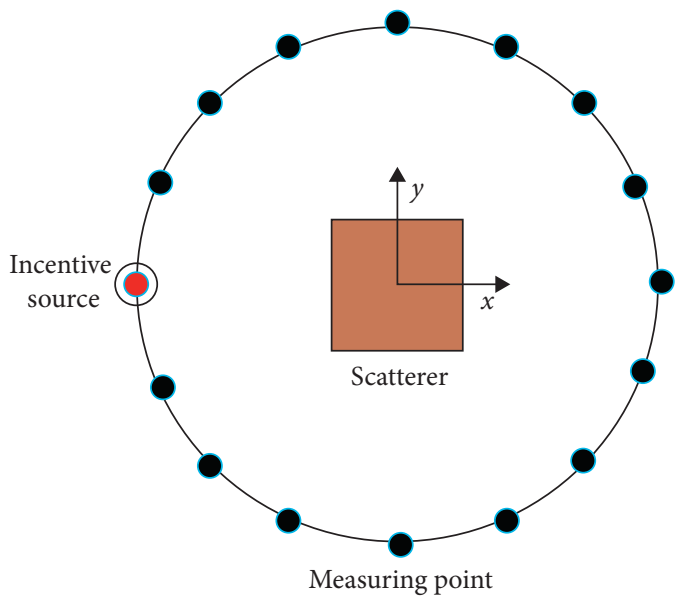

Figure 6: The model of a scattered field.

TABLE 6: The results of the algorithm performance comparison.

\begin{tabular}{|c|c|c|c|c|}
\hline Algorithm & Max & Min & $f_{\text {min }}$ & MSE \\
\hline $\mathrm{DE}$ & 4.3225 & 2.3503 & $4.6410 \times 10^{-7}$ & 0.2310 \\
\hline PSO & 3.7381 & 1.4923 & 0.0072 & 0.3084 \\
\hline SCA & 4.4657 & 1.0000 & 0.0511 & 0.8155 \\
\hline $\begin{array}{l}\text { Proposed } \\
\text { algorithm }\end{array}$ & 3.0007 & 2.9995 & $9.5047 \times 10^{-6}$ & $1.1017 \times 10^{-7}$ \\
\hline \multicolumn{5}{|c|}{$\begin{array}{l}\text { The number of iterations } 1000 \\
\text { The number of populations } 50\end{array}$} \\
\hline
\end{tabular}

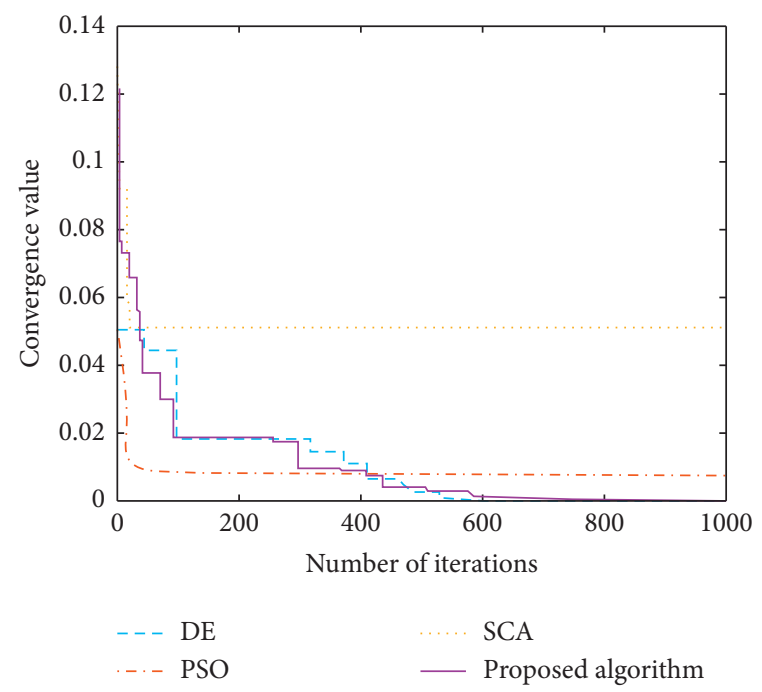

Figure 7: Convergence curve.

According to the free-space wavelength, the imaging domain is divided into $N=10 \times 10$ subdomains.

To test the property of the proposed method, three other common algorithms (PSO, DE, and SCA) are used for comparison purposes. The number of iterations is 200 , and the population number is 50 . The results of the reconstruction are shown in Figure 9.

The convergence curve is shown in Figure 10. 


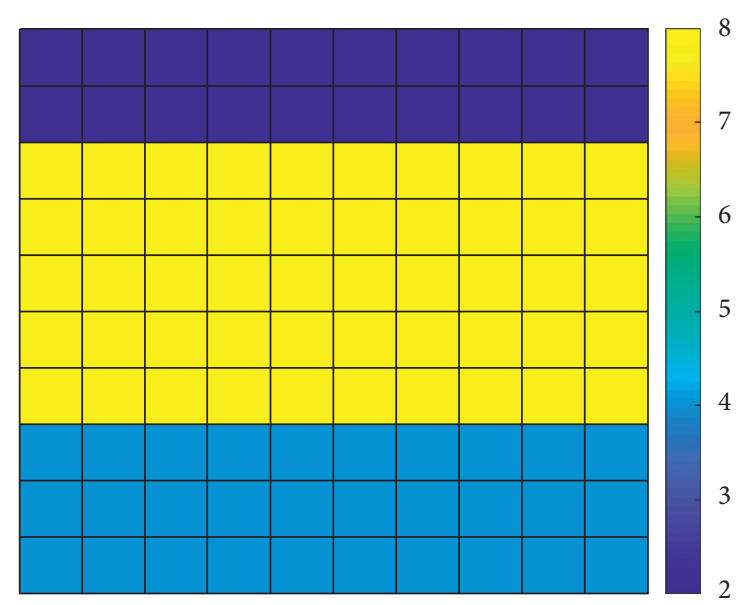

FIGURE 8: Relative permittivity distribution of the layered permittivity distribution.

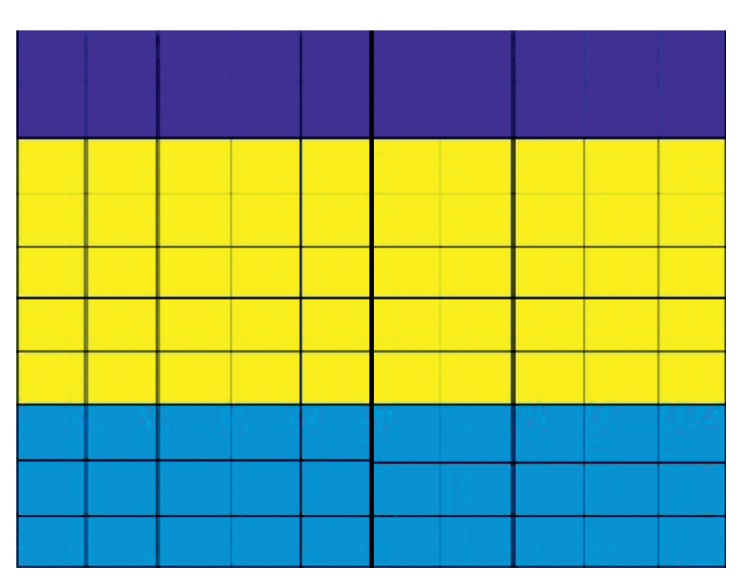

(a)

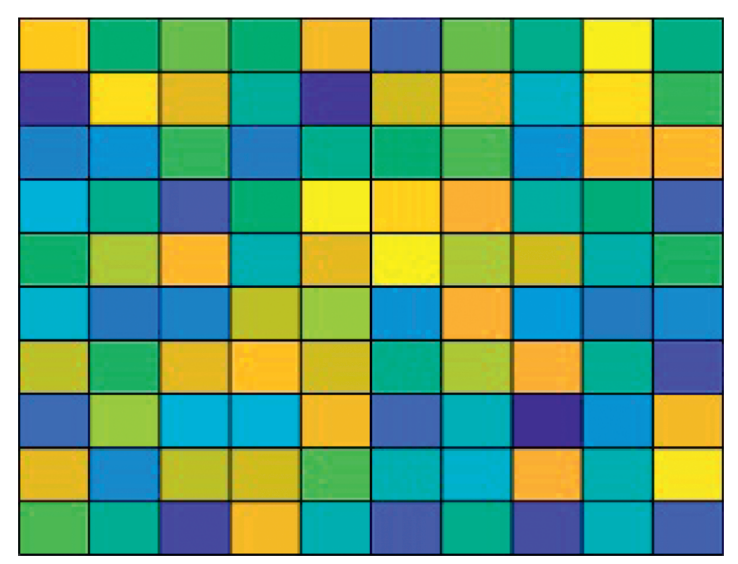

(c)
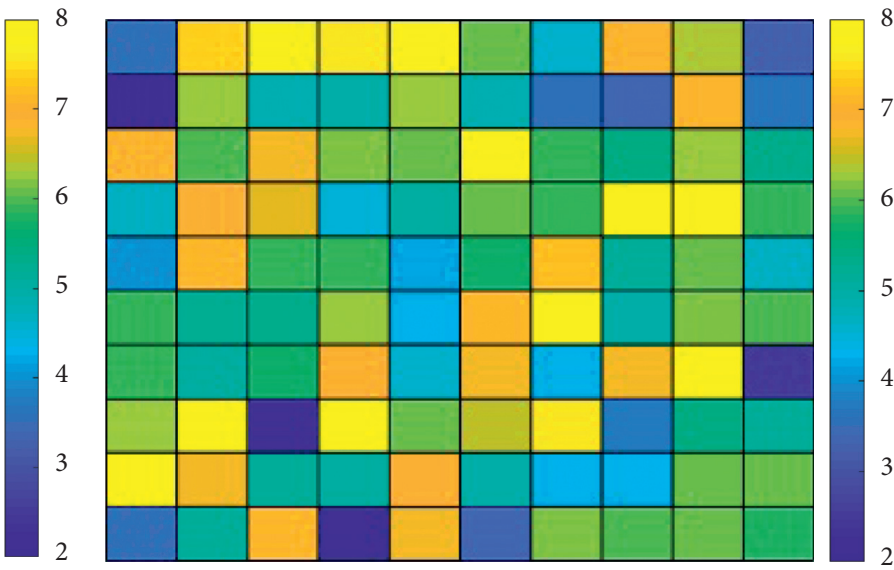

(b)

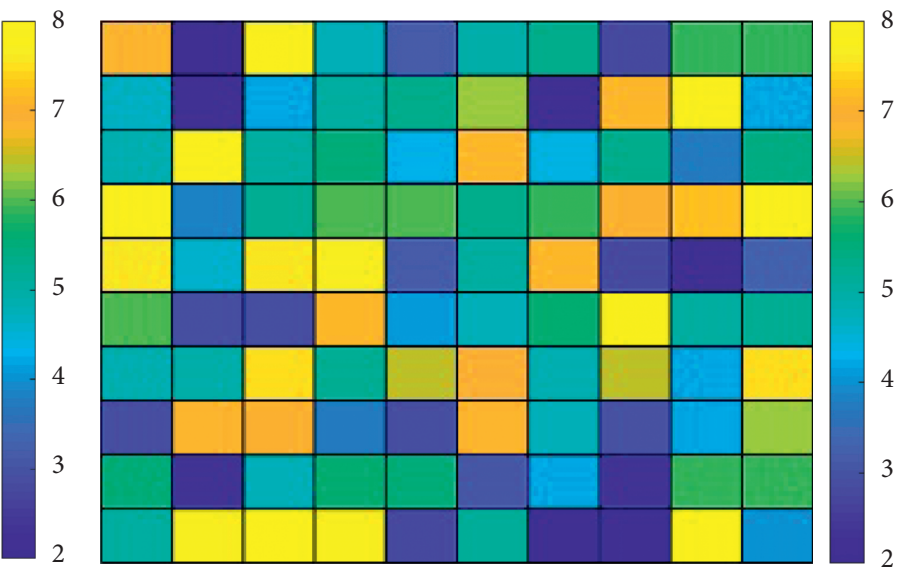

(d)

Figure 9: Reconstruction result of different algorithms. (a) Proposed algorithm. (b) PSO. (c) DE. (d) SCA. 


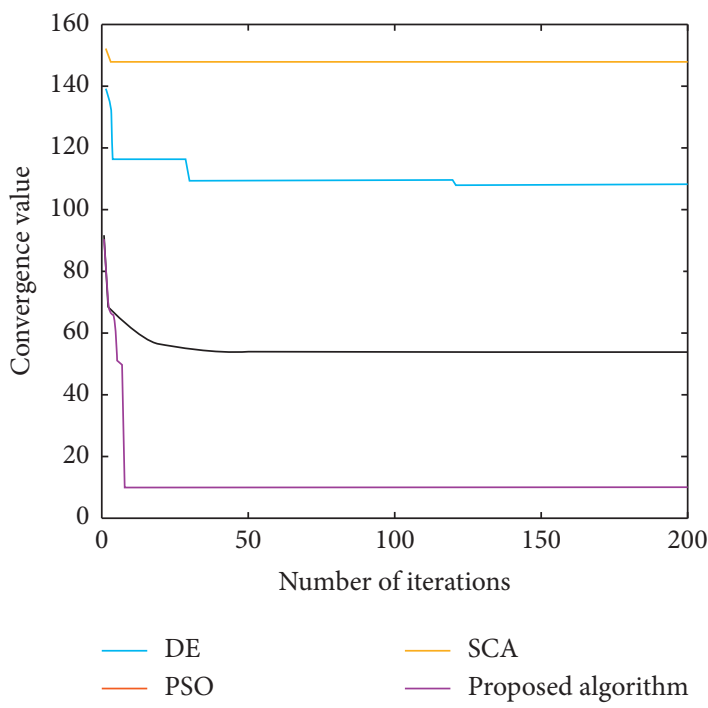

FIgURE 10: Convergence curve.

TABLE 7: The correlation coefficient between the obtained scattered field data and the original field data.

\begin{tabular}{lcccc}
\hline Algorithm & PSO & DE & SCA & Proposed HA \\
\hline Original field data & 0.9307 & 0.6387 & 0.7241 & $\mathbf{0 . 9 7 0 0}$ \\
\hline
\end{tabular}

Based on the results of convergence curve, the four methods all have reconstruction errors. This is due to the serious morbidity of the inverse scattering problem. Obviously, the four algorithms have fallen into different degrees of precocity. However, the convergence value of the proposed algorithm is the smallest.

From the imaging results, the three other optimization algorithms cannot reconstruct the number of layers and distribution of the permittivity of the scatterer well. The nature of the scatterer cannot be effectively observed from the results. The proposed algorithm possesses the ability to reconstruct the stratification of the permittivity distribution.

Correlation coefficient is a statistical indicator, which is used to reflect the close degree of correlation between variables. According to the reconstruction results, the correlation coefficient between the scattered field data obtained by optimization and original field data is shown in Table 7 .

It can be seen from the correlation coefficient that the correlation coefficient obtained by the proposed algorithm is the largest among the listed algorithm, that is, the reconstructed scattered field data are closest to the original field data.

4.6. Reconstruction of Materials with Bubbles. As an example, consider a problem of reconstructing a scatterer with bubbles in it. The shape of the scatterer is the same as the above part. The permittivity equals 5 . Three bubbles are randomly distributed in the scatterer, as shown in Figure 11.

Four incentive sources with a frequency of $2 \mathrm{GHz}$ are adopted, which is $0.1 \mathrm{~m}$ from the center of the scatterer. Sixty

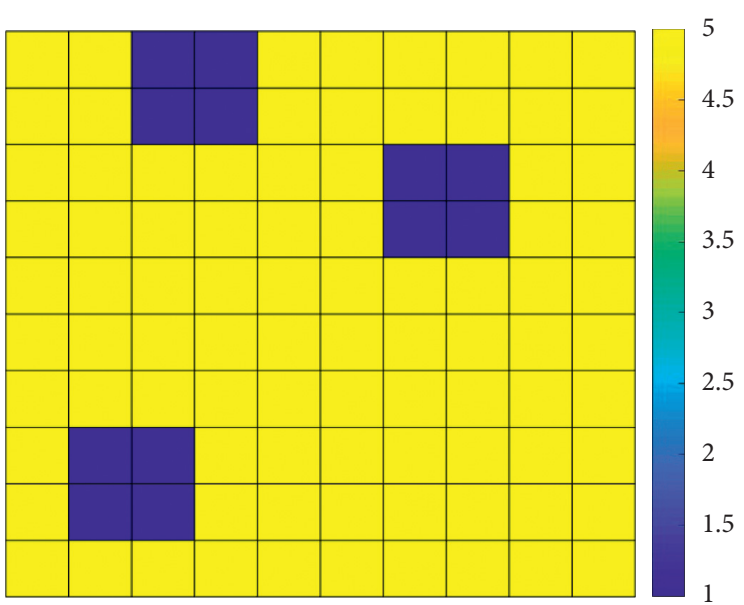

FIGURE 11: Relative permittivity distribution of materials with bubbles.

receiving points are evenly applied on a circle. The radius of circle is $1.5 \mathrm{~m}$. According to the free-space wavelength, the imaging domain is partitioned into $N=10 \times 10$ subdomains.

To test the properties of the proposed method, three other common algorithms (PSO, DE, and SCA) are used for comparison purposes. The number of iterations is 200 , and the population number is 50 . The reconstruction results are illustrated in Figure 12.

The convergence curve is shown in Figure 13.

From the results, the four methods all have reconstruction errors, but the convergence value of the proposed 


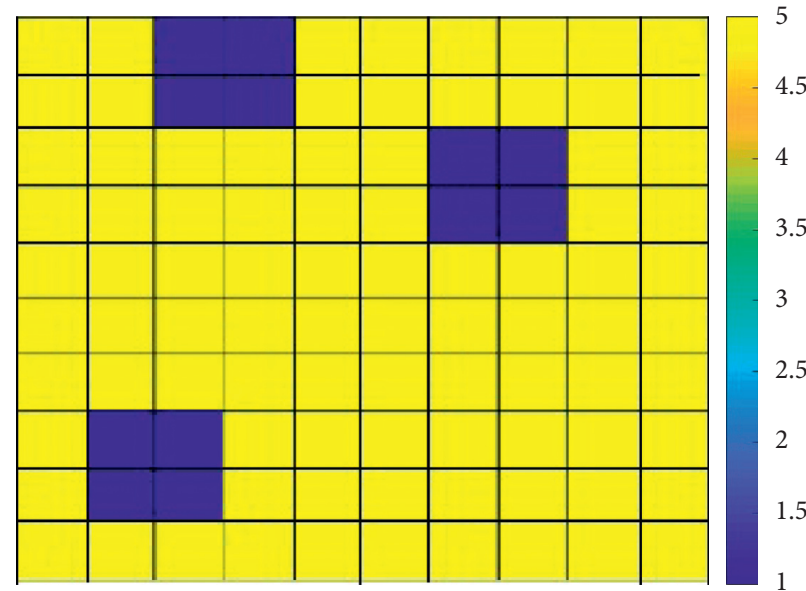

(a)

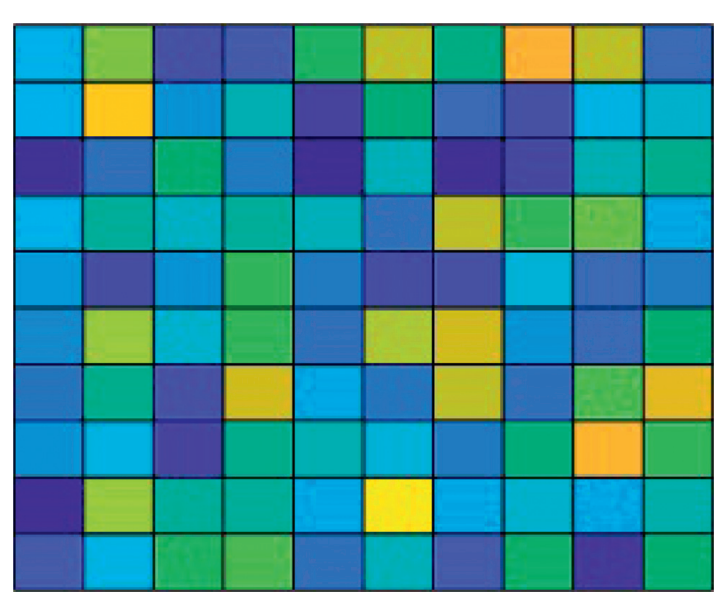

(c)

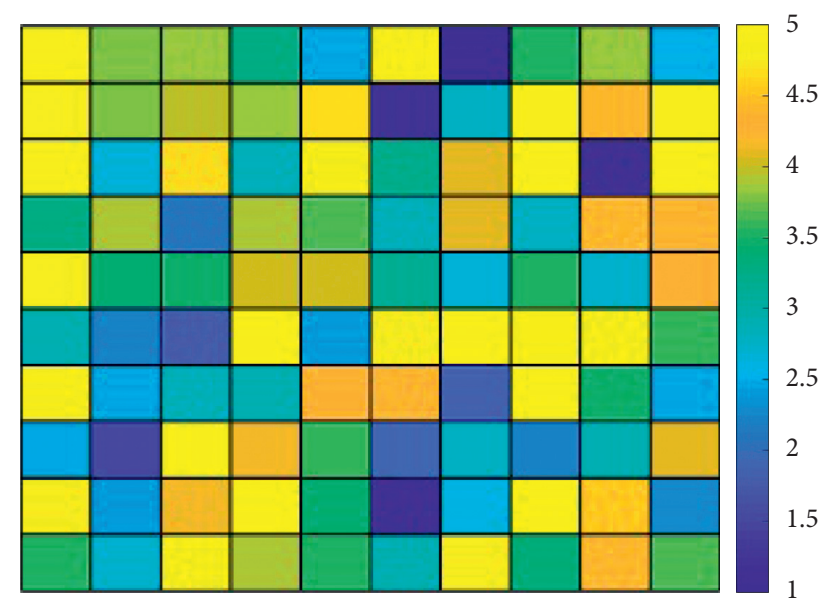

(b)
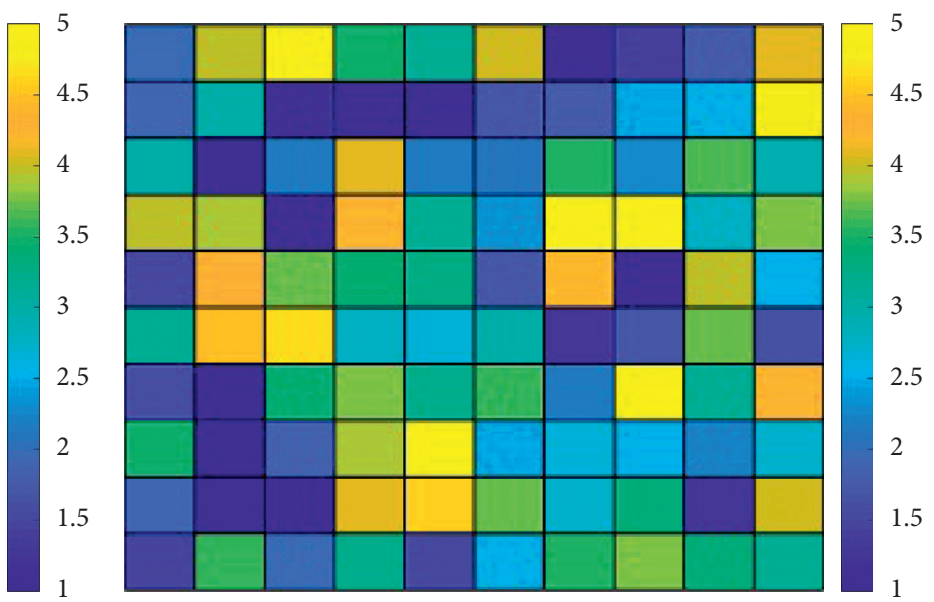

(d)

Figure 12: Reconstruction result of the different algorithms. (a) Proposed algorithm. (b) PSO. (c) DE. (d) SCA.

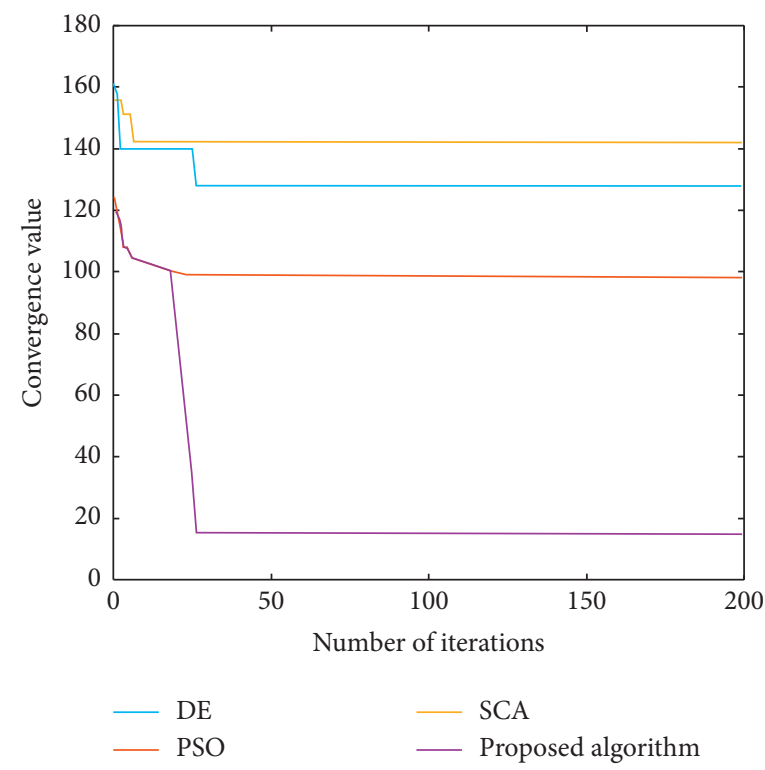

Figure 13: Convergence curve. 
TABLE 8: The correlation coefficient between the obtained scattered field data and the original field data.

\begin{tabular}{lcccc}
\hline Algorithm & PSO & DE & SCA & Proposed HA \\
\hline Original field data & 0.5281 & 0.4854 & 0.5253 & $\mathbf{0 . 9 5 0 7}$ \\
\hline
\end{tabular}

algorithm is the smallest. It can be observed that the three other optimization algorithms cannot reconstruct the bubble positions and the permittivity of the scatterer. The reconstruction result cannot reflect the material properties of the scatterer and bubble positions well. The proposed algorithm possesses the ability of reconstruction for materials with bubbles.

The correlation coefficient between the obtained scattered field data and the original field data is shown in Table 8:

It is shown that the correlation coefficient obtained by the proposed algorithm HA is the largest among the listed algorithm. It means that the reconstructed scattered field data obtained by the proposed algorithm are closest to the original field data.

It can be seen from quantitative analysis results that the proposed hybrid algorithm, when solving reconstruction of the uniform permittivity distribution, can obtain the smaller resulting error and more stable solution than the traditional stochastic optimization algorithm listed. And, it can be seen from qualitative analysis results that when solving the reconstruction of layered permittivity distribution and of materials with bubbles, the proposed algorithm is proved to be able to solve these problems.

\section{Conclusion}

In this paper, a hybrid algorithm (HA) based on a modified SCA and least square is proposed to solve the microwave imaging problems.

Firstly, the proposed algorithm improves the search ability of traditional SCA. The search path of traditional algorithms has been modified. The improved algorithm can obtain the optimal solution closer to the theoretical value.

Then, to be more suitable for diverse and complicated microwave imaging problems, the least square method is introduced to form a joint and parallel algorithm. Numerical results show that the hybrid algorithm is better applicable for solving the problem of complex scatterer imaging.

In addition, two classical engineering design problems are applied to prove the properties of the proposed algorithm. The experiment results demonstrated the validity and efficiency of the HA, which can get the minimum cost compared with the results from the references.

However, at the same time, the inverse scattering problem used in the paper has relatively small dimensions and does not involve lossy materials, that is, the permittivity of the scatterer has a large imaginary part. Therefore, in the future research, the research direction of the physical-inspired algorithm for lossy materials and high-dimensional inverse scattering should be explored.

\section{Data Availability}

The data used to support the findings of this study are available from the corresponding author upon request.

\section{Conflicts of Interest}

The authors declare that they have no conflicts of interest.

\section{Acknowledgments}

The authors would like to thank the journal editor for the work done for the manuscript sincerely.

\section{References}

[1] E. J. Bond, L. Xu Li, S. C. Hagness, and B. D. Van Veen, "Microwave imaging via space-time beamforming for early detection of breast cancer," IEEE Transactions on Antennas and Propagation, vol. 51, no. 8, pp. 1690-1705, 2003.

[2] D. Tajik, J. Trac, and N. K. Nikolova, "Quality control of microwave equipment for tissue imaging," IEEE Journal of Electromagnetics, RF and Microwaves in Medicine and Biology, vol. 4, no. 1, pp. 52-60, 2020.

[3] Y. Zhang, H. Liu, J. Wu, C. Zhang, and J. He, "Method to reduce imaging errors in dynamic target observation of the geostationary interferometric microwave sounder," IEEE Geoscience and Remote Sensing Letters, vol. 14, no. 2, pp. 267-271, 2017.

[4] M. Pastorino, "Stochastic optimization methods applied to microwave imaging: a review," IEEE Transactions on Antennas and Propagation, vol. 55, no. 3, pp. 538-548, 2007.

[5] J. Kennedy and R. Eberhart, "Particle swarm optimization," in Proceedings of the ICNN'95-International Conference on Neural Networks, pp. 1942-1948, Perth, Australia, 1995.

[6] S. Das and P. N. Suganthan, "Differential evolution: a survey of the state-of-the-art," IEEE Transactions on Evolutionary Computation, vol. 15, no. 1, pp. 4-31, 2011.

[7] J. L. F. Martínez and E. García Gonzalo, "PSO advances and application to inverse problems," in Swarm, Evolutionary, and Memetic Computing, Lecture Notes in Computer ScienceSpringer, Berlin, Germany, 2010.

[8] M. Donelli, G. Franceschini, A. Martini, and A. Massa, "An integrated multiscaling strategy based on a particle swarm algorithm for inverse scattering problems," IEEE Transactions on Geoscience and Remote Sensing, vol. 44, no. 2, pp. 298-312, 2006.

[9] A. Semnani, M. Kamyab, and I. T. Rekanos, "Reconstruction of one-dimensional dielectric scatterers using differential evolution and particle swarm optimization," IEEE Geoscience and Remote Sensing Letters, vol. 6, no. 4, pp. 671-675, 2009.

[10] C.-C. Chiu, C.-H. Sun, C.-L. Li, and C.-H. Huang, "Comparative study of some population-based optimization algorithms on inverse scattering of a two-dimensional perfectly conducting cylinder in dielectric slab medium," IEEE Transactions on Geoscience and Remote Sensing, vol. 51, no. 4, pp. 2302-2315, 2013.

[11] Y.-T. Cheng, C.-C. Chiu, S.-P. Chang, and J.-C. Hsu, "Comparison of particle swarm optimization and self-adaptive dynamic differential evolution for the imaging of a periodic conductor," International Journal of Applied Electromagnetics and Mechanics, vol. 46, no. 1, pp. 69-79, 2014. 
[12] T. Huang and A. Sanagavarapu Mohan, "A microparticle swarm optimizer for the reconstruction of microwave images," IEEE Transactions on Antennas and Propagation, vol. 55, no. 3, pp. 568-576, 2007.

[13] A. Semnani, I. T. Rekanos, M. Kamyab, and T. G. Papadopoulos, "Two-dimensional microwave imaging based on hybrid scatterer representation and differential evolution," IEEE Transactions on Antennas and Propagation, vol. 58, no. 10, pp. 3289-3298, 2010.

[14] L. Yang, "An improved evolution strategy and its application to inverse scattering in microwave imaging," IEEE Transactions on Magnetics, vol. 51, no. 3, pp. 1-4, 2015.

[15] C. Yang, J. Zhang, and M. Tong, "Solving inverse scattering problems through ba-based optimization," in Proceedings of the 2019 Photonics \& Electromagnetics Research Symposium-Fall (PIERS_Fall), Xiamen, China, December 2019.

[16] K.-C. Lee and P.-T. Lu, "Application of whale optimization algorithm to inverse scattering of an imperfect conductor with corners," International Journal of Antennas and Propagation, vol. 2020, Article ID 8205797, 9 pages, 2020.

[17] R. Salgotra and U. Singh, "The naked mole-rat algorithm," Neural Computing and Applications, vol. 31, no. 12, pp. 8837-8857, 2019.

[18] S. Mirjalili, "SCA: a Sine Cosine Algorithm for solving optimization problems," Knowledge-Based Systems, vol. 96, pp. 120-133, 2016.

[19] A. A. Ewees, M. Abd Elaziz, M. A. A. Al-Qaness, H. A. Khalil, and S. Kim, "Improved artificial bee colony using sine-cosine algorithm for multi-level thresholding image segmentation," IEEE Access, vol. 8, pp. 26304-26315, 2020.

[20] T. Guesmi, A. Farah, I. Marouani, B. Alshammari, and H. H. Abdallah, "Chaotic sine-cosine algorithm for chanceconstrained economic emission dispatch problem including wind energy," IET Renewable Power Generation, vol. 14, no. 10, pp. 1808-1821, 2020.

[21] W. Fu, K. Shao, J. Tan, and K. Wang, "Fault diagnosis for rolling bearings based on composite multiscale fine-sorted dispersion entropy and SVM with hybrid mutation SCAHHO algorithm optimization," IEEE Access, vol. 8, pp. 13086-13104, 2020.

[22] N. A. Mhd Rizal, M. Falfazli Mat Jusof, A. A. Abd Razak, S. Mohammad, and A. N. Kasruddin Nasir, "Spiral sine-cosine algorithm for global optimization," in Proceedings of the 2019 IEEE 9th Symposium on Computer Applications \& Industrial Electronics (ISCAIE), pp. 234-238, Malaysia, Malaysia, 2019.

[23] M. A. A. Al-qaness, M. A. Elaziz, and A. A. Ewees, "Oil consumption forecasting using optimized adaptive neurofuzzy inference system based on sine cosine algorithm," IEEE Access, vol. 6, pp. 68394-68402, 2018.

[24] N. Nayak, S. Mishra, D. Sharma, and B. Kumar Sahu, “Application of modified sine cosine algorithm to optimally design PID/fuzzy-PID controllers to deal with AGC issues in deregulated power system," IET Generation, Transmission \& Distribution, vol. 13, no. 12, pp. 2474-2487, 2019.

[25] A. Banerjee and M. Nabi, "Re-entry trajectory optimization for space shuttle using Sine-Cosine Algorithm," in Proceedings of the 2017 8th International Conference on Recent Advances in Space Technologies (RAST), pp. 73-77, Istanbul, Turkey, June 2017.

[26] A. Ramadan, M. Ebeed, and S. Kamel, "Performance assessment of a realistic egyptian distribution network including PV penetration with DSTATCOM," in Proceedings of the 2019 International Conference on Innovative Trends in Computer
Engineering (ITCE), pp. 426-431, IEEE, Aswan City, Egypt, February 2019.

[27] S. A. M. Abdelwahab, A. M. Yousef, M. Ebeed, F. K. AboElyousr, A. Elnozohy, and M. Mohamed, "Optimization of PID controller for hybrid renewable energy system using adaptive sine cosine algorithm," International Journal of Renewable Energy Research (IJRER), vol. 10, no. 2, pp. 669677, 2020.

[28] S. Abdel-Fatah, M. Ebeed, and S. Kamel, "Optimal reactive power dispatch using modified sine cosine algorithm," in Proceedings of the 2019 International Conference on Innovative Trends in Computer Engineering (ITCE), pp. 510-514, IEEE, Aswan, Egypt, February 2019.

[29] R. C. Eberhart and J. Kennedy, "A new optimizer using particle swarm theory," in Proceedings of the Sixth International Symposium on Micro Machine and Human Science, pp. 39-43, Nagoya, Japan, October 1995.

[30] X. Chen, Computational Methods for Electromagnetic Inverse Scattering, John Wiley \& Sons, Hoboken, NJ, USA, 2018.

[31] J. J. Jamian, M. N. Abdullah, H. Mokhlis, M. W. Mustafa, and A. H. A. Bakar, "Global particle swarm optimization for high dimension numerical functions analysis," Journal of Applied Mathematics, vol. 2014, Article ID 329193, 14 pages, 2014.

[32] G. Lu and Z. Cao, "Radiation pattern synthesis with improved high dimension PSO," in Proceedings of the 2017 Progress in Electromagnetics Research Symposium-Fall (PIERS-FALL), pp. 2160-2165, Singapore, Singapore, November 2017.

[33] S. Mirjalili, S. M. Mirjalili, and A. Lewis, "Grey wolf optimizer," Advances in Engineering Software, vol. 69, no. 3, pp. 46-61, 2014.

[34] S. Mirjalili, "Moth-flame optimization algorithm: a novel nature-inspired heuristic paradigm," Knowledge-Based Systems, vol. 89, pp. 228-249, 2015.

[35] W. Long, "Solving high-dimensional global optimization problems using an improved sine cosine algorithm," Expert Systems with Applications, vol. 123, 2018. 